\title{
Prevalencia e intensidad de la infección por geohelmintos y prevalencia de la malaria en escolares de El Salvador
}

\author{
Óscar René Sorto', Alexandra Manoella Portillo², Miguel Ángel Aragón³, Martha Idalí Saboyá4, \\ María Paz Ade ${ }^{5}$, Miguel Ángel Minero ${ }^{3}$, Marta Alicia Hernández ${ }^{6}$, Amada Gloria Mena ${ }^{6}$, \\ Rodolfo Peña ${ }^{3}$, Victor Manuel Mejía ${ }^{3}$, Keith Carter ${ }^{5}$
}

1 Dirección de Vigilancia Sanitaria, Ministerio de Salud, San Salvador, El Salvador

2 Programa de Enfermedades Infecciosas Desatendidas, Dirección de Enfermedades Infecciosas, Ministerio de Salud, San Salvador, El Salvador

${ }^{3}$ Organización Panamericana de la Salud, Representación de El Salvador, San Salvador, El Salvador

${ }^{4}$ Programa de Enfermedades Infecciosas Desatendidas, Organización Panamericana de la Salud-Organización Mundial de la Salud, Washington, D.C., Estados Unidos

5 Programa Regional de Malaria, Organización Panamericana de la Salud-Organización Mundial de la Salud, Washington, D.C., Estados Unidos

${ }^{6}$ Laboratorio Nacional de Referencia, Ministerio de Salud, San Salvador, El Salvador

Introducción. El Salvador no cuenta con datos actualizados de prevalencia en menores de 15 años de la infección por helmintos transmitidos por contacto con el suelo. Además, al ser uno de los países en las Américas que reporta un número bajo de casos de malaria, se considera que su eliminación allí es factible.

Objetivo. Determinar la prevalencia y la intensidad de la infección por geohelmintos y la prevalencia de Plasmodium spp. en escolares de 8 a 10 años de El Salvador.

Materiales y métodos. Se hizo un estudio de corte transversal en las cinco zonas eco-epidemiológicas del país (planicie costera, depresión central, cadena volcánica, cadena costera y zona montañosa). La presencia de geohelmintiasis se estudió en 1.325 estudiantes y, la de malaria, en 152. Se utilizó la técnica de Kato-Katz para la detección de geohelmintos, y para el diagnóstico de la malaria, una prueba rápida, el estudio mediante microscopía y la prueba de reacción en cadena de la polimerasa.

Resultados. La prevalencia total de geohelmintiasis fue de 7,9\% (IC $\left.{ }_{95 \%} 6,6-9,5\right)$. En la planicie costera fue de 14,9\% (IC $\left.{ }_{95 \%} 10,9-19,7\right)$; en la depresión central, de 9,4\% ( $\left.\mathrm{IC}_{95 \%} 6,5-13,3\right)$; en la cadena volcánica, de $6,6 \%\left(\mathrm{IC}_{95 \%} 4,2-10,5\right)$; en la cadena costera, de 5,9\% (IC $\left.{ }_{95 \%} 3,8-9,4\right)$, y en la cadena montañosa, de $2,6 \%\left(\mathrm{IC}_{95 \%}\right.$ 1,4-5,7). La proporción de infección de gran intensidad debida a cualquiera de las especies de geohelmintos fue de $0,3 \%$. No se encontraron escolares infectados con Plasmodium spp.

Conclusión. La prevalencia de geohelmintos fue baja y la especie más prevalente fue Trichuris trichiura. La intensidad de la infección debida a cualquiera de las especies de geohelmintos fue leve $(<1 \%)$. Los factores de riesgo asociados a la infección por geohelmintos fueron la defecación al aire libre, no usar calzado y vivir en la planicie costera.

Palabras clave: helmintos, helmintiasis, malaria, Ascaris, Trichuris, infecciones por uncinaria, prevalencia, El Salvador.

doi: http://dx.doi.org/10.7705/biomedica.v35i3.2408

Prevalence and intensity of infection by soil-transmitted helminths and prevalence of malaria among schoolchildren in El Salvador

Introduction: El Salvador does not have recent data on the prevalence of infection with soil-transmitted helminths among children aged under 15 years of age. As one of the countries in the Americas that reports few malaria cases, eradication of this disease from El Salvador is considered to be feasible.

Objective: To determine the prevalence and intensity of infection by soil-transmitted helminths, as well as the prevalence of Plasmodium spp. in schoolchildren aged 8-10.

Materials and methods: A cross-sectional study was carried out in each of the five eco-epidemiological zones of the country (coastal plain, central basin, volcanic range, coastal range and mountain zone). In all 1,325 students we studied the presence of geohelminthiasis, with 152 of them also being tested

\footnotetext{
Contribución de los autores:

Óscar René Sorto, Alexandra Manoella Portillo, Miguel Ángel Aragón, Miguel Ángel Minero, Marta Alicia Hernández y Amada Gloria Mena: participación en el trabajo de campo

Todos los autores participaron en el desarrollo del protocolo, el análisis de la información y la elaboración del artículo científico.
} 
for malaria. The Kato-Katz technique was used to detect geohelminths while diagnosis of malaria was performed using the rapid diagnostic test, microscopy and polymerase chain reaction.

Results: The overall prevalence of geohelminthiasis was $7.9 \%(95 \% \mathrm{Cl} 6.6-9.5 \%)$. Values for the five eco-epidemiological zones were as follows: coastal plain, $14.9 \%(95 \% \mathrm{Cl} 10.9-19.7 \%)$; central plateau, $9.4 \%(95 \% \mathrm{Cl} 6.5-13.3 \%)$; volcanic range, 6.6\% (95\%Cl 4.2-10.5\%); coastal range, 5.9\% (95\%Cl 3.8$9.4 \%)$, and mountain zone, $2.6 \%(95 \% \mathrm{Cl} 1.4-5.7 \%)$. The overall rate of high intensity infection with any of the geohelminth species was $0.3 \%$. No schoolchildren were found infected with Plasmodium spp. by any of the three diagnostic techniques used.

Conclusion: Prevalence of geohelminths was low and Trichuris trichiura was the predominant species. Intensity of infection with any of the species of geohelminths was light $(<1 \%)$. The risk factors associated with infection by soil-transmitted helminths were defecation in the open air, being barefoot and living in coastal areas.

Key words: Helmints, helminthiases, malaria; Ascaris, Trichuris, hookworm infections, prevalence, El Salvador.

doi: http://dx.doi.org/10.7705/biomedica.v35i3.2408

Las enfermedades infecciosas desatendidas continúan teniendo un gran impacto negativo en grandes segmentos de la población, especialmente en los países en desarrollo (1). El impacto nacional y regional de estas enfermedades se mide en términos de disminución de la productividad, agravamiento de la pobreza, desarrollo socioeconómico lento, deterioro de la calidad de vida y, a la larga, una mayor necesidad de inversión pública para el manejo y el tratamiento de las secuelas que producen.

En la Resolución CD49.R19 aprobada en el marco del Consejo Directivo del 2009, los estados miembro de la Organización Panamericana de la SaludOrganización Mundial de la Salud (OPS/OMS) acogieron la meta de eliminar las enfermedades desatendidas y otras infecciones relacionadas con la pobreza, entre ellas, las infecciones por geohelmintos (Ascaris lumbricoides, Trichuris trichiura y uncinarias) y la malaria. Específicamente, se incluyó el objetivo de reducir la prevalencia de las geohelmintiasis en niños en edad escolar en áreas de alto riesgo, de más de $50 \%$ a menos de $20 \%$ (2). Las estrategias recomendadas para lograrlo son la administración regular del tratamiento preventivo o la distribución masiva de medicamentos para, al menos, $75 \%$ de los niños en edad escolar en riesgo de infección según el nivel de prevalencia en cada país, así como la promoción del acceso a agua potable, saneamiento básico y educación en salud mediante el trabajo intersectorial. La meta de eliminación de la malaria

\section{Correspondencia:}

Miguel Ángel Aragón, Organización Panamericana de la Salud, Representación El Salvador, 73 Avenida Sur, \#135, Colonia Escalón, apartado postal 1072, San Salvador, El Salvador Teléfono: 00503-2511-9517

aragonm@paho.org

Recibido: 05/06/14; aceptado: 08/05/15 se estableció en las áreas en donde la interrupción de la transmisión local fuera factible, incluidos los países de Centroamérica (3).

Según los datos del Programa Regional de Enfermedades Infecciosas Desatendidas de la OPS/OMS en el 2006, ocho de los 35 países de Latinoamérica habían completado los estudios de prevalencia a nivel nacional (Argentina, Belice, Brasil, Haití, Honduras, México, Nicaragua y Venezuela); de estos países, solo México y Venezuela habían reportado una prevalencia menor de $20 \%$. En el 2009, por lo menos, 27 países necesitaban actualizar los datos mediante un nuevo mapeo, o iniciar sus estudios sobre la prevalencia y la intensidad de la infección (1).

En el 2013, la OPS/OMS publicó los datos de prevalencia e intensidad de la infección por geohelmintos de los 18 países de Latinoamérica y el Caribe con datos publicados entre el 2000 y el 2010 (Argentina, Belice, Bolivia, Brasil, Colombia, Costa Rica, Cuba, Ecuador, Guatemala, Guyana, Haití, Honduras, México, Nicaragua, Paraguay, Perú, Santa Lucía y Venezuela), lo que evidenció una brecha importante de información en 12 de los 30 países endémicos para las geohelmintiasis en la región (4), entre los cuales se encuentra El Salvador. Dicha situación constituye una deuda cuyo pago es inaplazable, especialmente en la población escolar en riesgo, estimada en casi medio millón de niños en edad preescolar, y en 1,5 millones y 1,4 millones de niños en edad escolar entre el 2005 y el 2009 por el Ministerio de Salud con base en el censo del Ministerio de Educación (5).

Según los cálculos de la OPS/OMS, en El Salvador hay 233.387 niños entre 1 y 14 años en riesgo de sufrir infección por geohelmintos. Estas cifras se calculan cada año aplicando el algoritmo publicado 
por la OMS en el 2009 con base en el indicador sustituto de acceso a instalaciones mejoradas de saneamiento básico para la identificación de poblaciones en riesgo cuando no se dispone de los datos de prevalencia (6).

En relación con la prevención, el control y la posible eliminación de la malaria, en la Resolución CD51/11 del 2011 (7), la OPS/OMS instó a los estados miembro a que revisaran los planes nacionales o establecieran otros nuevos, empleando un enfoque que abordara los factores sociales determinantes de la salud, y previera la colaboración entre programas y sectores para reducir el endemismo y avanzar hacia la eliminación donde fuera factible.

Según el informe global de la situación del paludismo del 2013, El Salvador estaba en la etapa previa a la eliminación, pues había reportado 13 casos autóctonos y seis importados, lo que significaba una reducción de la incidencia de más de $75 \%$ en comparación con lo reportado en el año 2000 (8). Debido a esta disminución de casos y a la vigilancia activa, era necesario saber si en las zonas endémicas con un número muy bajo de casos existían casos asintomáticos de malaria no identificados en la vigilancia rutinaria, que implicaran riesgo de brotes. En ese sentido, el Ministerio de Salud, con apoyo de la OPS/OMS, decidió hacer la presente encuesta de prevalencia e intensidad de la infección por geohelmintos en escolares de 8 a 10 años de edad, aprovechando la oportunidad para evaluar también la prevalencia de la malaria y detectar obstáculos para el cumplimiento de la meta de eliminación de la enfermedad.

\section{Materiales y métodos}

\section{Universo y población de estudio}

El universo del estudio fue la población nacional de escolares entre los 5 y los 14 años de edad, es decir, el grupo más afectado por la infección con geohelmintos. Se seleccionó el grupo de niños entre los 8 y los 10 años de edad (tercer grado de primaria), pues representan adecuadamente la situación de la prevalencia y la intensidad de la infección por geohelmintos en escolares (9). Se incluyeron 154.231 estudiantes de ambos sexos matriculados en el tercer grado de primaria en 5.454 centros escolares públicos y privados de El Salvador, según los datos del Ministerio de Educación en el 2010 (5).

\section{Tipo de estudio}

Se llevó a cabo un estudio de corte transversal en varias etapas, estratificado según las cinco zonas eco-epidemiológicas del país. Se hizo una selección aleatoria simple de los municipios (conglomerados) y de los centros escolares. La selección de los municipios fue proporcional al tamaño de la población y respondió a la presencia de los llamados equipos comunitarios de salud familiar, dado el apoyo logístico que estos podían brindar. No se consideró la estratificación por áreas rurales y urbanas.

\section{Tamaño de la muestra}

Con base en los lineamientos de la OPS/OMS (10), el tamaño adecuado y representativo de la muestra se estableció por zonas eco-epidemiológicas homogéneas: en cada una de ellas se estableció un tamaño de muestra de 200 a 250 niños, sin ajuste adicional por efecto del diseño. Se agregó $12 \%$ para ajustar por posibles pérdidas, para un total de 280 niños por zona y 1.400 a nivel nacional. Puesto que la mayor proporción de la población se concentraba en el municipio de San Salvador (capital del país), se tomó este como un conglomerado especial y se muestreó una escuela adicional, completando 1.428 niños.

\section{Muestreo}

Estratos y entidades geográficas. Se utilizaron las zonas eco-epidemiológicas establecidas por el Servicio Nacional de Estudios Territoriales del Ministerio de Medio Ambiente y Recursos Naturales, como base de la estratificación (figura 1), pues aunque el territorio nacional es pequeño en extensión $\left(21.040,79 \mathrm{~km}^{2}\right)$, existen variantes en las condiciones ecológicas que podrían ejercer alguna influencia en la transmisión de los agentes en estudio, especialmente de los helmintos transmitidos por contacto con el suelo. Dichas zonas son la cadena costera, la planicie costera, la cadena volcánica, la depresión central y la zona montañosa fronteriza con Honduras. La distribución de los departamentos en las zonas eco-epidemiológicas se presenta en el cuadro 1.

Selección de los municipios. En cada zona ecológica se creó una base de datos de los municipios correspondientes. Las zonas se ordenaron de acuerdo con el total de la población municipal entre los 4 y los 17 años de edad, registrada en el Quinto Censo de Población y Vivienda de 2007. Con el fin de facilitar el operativo de campo, se hizo un listado de los municipios de cada zona eco-epidemiológica con presencia de equipos comunitarios de salud familiar, para capacitarlos y garantizar la recolección de las muestras y la medición de la talla y el peso de los niños. 


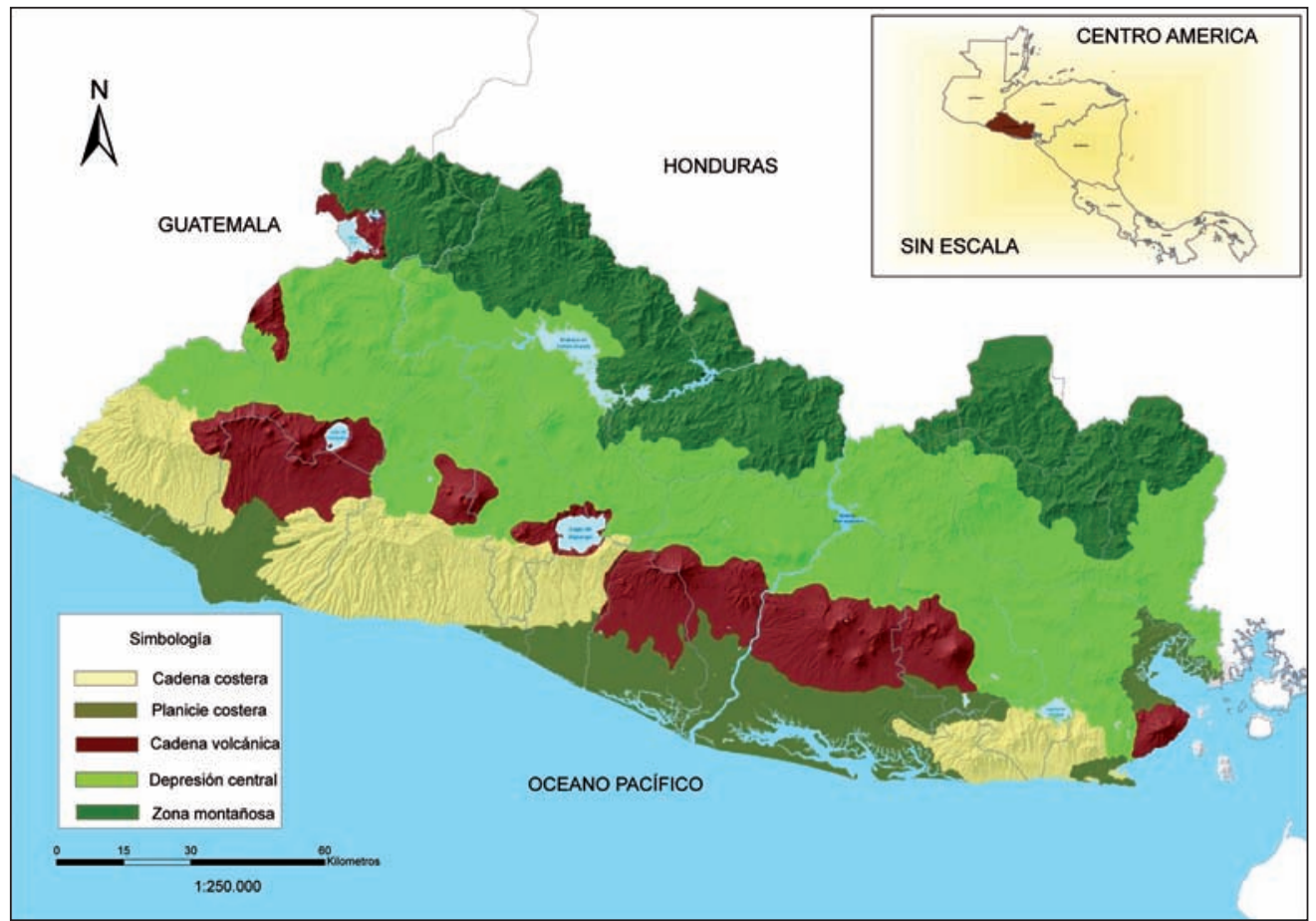

Figura 1. Distribución de zonas eco-epidemiológicas según el Servicio Nacional de Estudios Territoriales, 2002, El Salvador Adaptado de www.snet.gob.sv con autorización de la Dirección General del Observatorio Ambiental, Ministerio de Medio Ambiente y Recursos Naturales, 2014

Cuadro 1. Distribución de los departamentos de El Salvador por zonas eco-epidemiológicas para el estudio de prevalencia de la infección por geohelmintos y portadores de malaria en escolares, El Salvador, 2012

\begin{tabular}{|c|c|c|}
\hline No. & Zona & Departamentos o áreas de los departamentos incluidas en cada zona \\
\hline 1 & Cadena costera & $\begin{array}{l}\text { Centro de Ahuachapán, oriente de Sonsonate, sur de La Libertad y San Salvador, } \\
\text { occidente de La Paz, suroeste de Usulután, sur de San Miguel y La Unión }\end{array}$ \\
\hline 2 & Planicie costera & $\begin{array}{l}\text { Sur de Ahuachapán, Sonsonate, La Paz, San Vicente, Usulután y alrededores del } \\
\text { Golfo de Fonseca en La Unión }\end{array}$ \\
\hline 3 & Cadena volcánica & $\begin{array}{l}\text { Norte de Sonsonate, sur de Santa Ana, zona de El Boquerón y lago de llopango en } \\
\text { San Salvador, noroeste de La Paz, centro de San Vicente y Usulután, alrededores del } \\
\text { volcán de San Miguel y Conchagua en La Unión }\end{array}$ \\
\hline 4 & Depresión central & $\begin{array}{l}\text { Cuscatlán, norte de Ahuachapán, La Libertad, San Salvador, San Vicente, Usulután, } \\
\text { centro de Santa Ana y La Unión, centro norte de San Miguel, sur de Chalatenango } \\
\text { y Morazán. }\end{array}$ \\
\hline 5 & Zona montañosa & $\begin{array}{l}\text { Norte de Santa Ana, Morazán y La Unión; Cabañas y Chalatenango (excepto Nueva } \\
\text { Concepción) }\end{array}$ \\
\hline
\end{tabular}

Fuente: Ministerio de Medio Ambiente y Recursos Naturales, El Salvador

El número de 280 niños de la muestra en cada zona, se dividió por 30 , es decir, el promedio de estudiantes de tercer grado por escuela, para obtener el número de municipios (aproximadamente, 10 por zona eco-epidemiológica) y de escuelas participantes. Se hizo una selección aleatoria sistemática de los municipios por zona ecoepidemiológica proporcional al tamaño de la población. Por conveniencia se incluyeron cinco municipios del departamento de Ahuachapán que habían reportado focos de malaria en el último año (San Francisco Menéndez, Ahuachapán, Concepción de Ataco, Jujutla y Guaymango), en los cuales se tomaron muestras para detectar geohelmintos y malaria.

Selección de las escuelas y los niños. Se solicitó al Ministerio de Educación un listado de los centros escolares públicos de los municipios de la muestra, para seleccionar al azar un centro escolar. Considerando un promedio de 30 estudiantes de 
tercer grado por escuela, se incluyeron 28 niños con el fin de alcanzar el tamaño estimado de la muestra. En aquellos centros escolares donde el número de niños de tercer grado era inferior a 28, la muestra se completó con estudiantes en el rango de edad matriculados en segundo o cuarto grado. Los padres de los niños firmaron el consentimiento.

\section{Exámenes de laboratorio y recopilación de la información}

En todas las escuelas seleccionadas se recolectaron muestras de heces para la detección de geohelmintos y, en las de los cinco municipios con focos de malaria, se tomaron, además, muestras de sangre por punción dactilar. Las muestras de heces se procesaron mediante el método de KatoKatz (11), y la prevalencia y la intensidad de la infección por geohelmintos se determinaron entre las 24 y las 48 horas después de su recolección, con las fórmulas y rangos recomendados por la OMS (12).

Las muestras de sangre para el diagnóstico de malaria se distribuyeron así: dos alícuotas que cubrían $2 \mathrm{~cm}^{2}$ cada una en la parte central de un trozo de papel filtro (Whatman \#1) rotulado; una gota para el frotis de aproximadamente $5 \mu$ sobre una lámina portaobjeto de 3 pulgadas $\mathrm{x}, 1$ rotulada; dos gotas de $25 \mu \mathrm{l}$, aproximadamente, extendida cada una sobre una lámina portaobjetos, y una alícuota de $5 \mu$ para la prueba diagnóstica rápida de malaria (CareStart Malaria ${ }^{\circledR}$, Acces Bio, Inc. USA, pLDH/HRP2, P. vivax y P. falciparum).

Las muestras sanguíneas en papel de filtro y las láminas, se secaron a temperatura ambiente y se almacenaron, las primeras en bolsas plásticas individuales y las segundas en cajas porta-láminas. La prueba rápida se leyó en el sitio 20 minutos después de hacerla.

Las muestras de sangre en láminas y en papel de filtro se transportaron al Laboratorio Nacional de Referencia el mismo día de la recolección; las primeras se prepararon con coloración de Giemsa para la detección de Plasmodium spp. mediante microscopía y las segundas se enviaron al Laboratorio de Referencia Nacional de la Secretaría de Salud de Tegucigalpa, Honduras, para su análisis mediante PCR; el ADN se extrajo usando un estuche de Chelex-100 (Bio-Rad) (13) para la amplificación del gen 18Sr (14). Los productos de amplificación se observaron en geles de agarosa al $1 \%$ teñidos con bromuro de etidio.

\section{Medición de variables}

La información de edad y sexo de cada niño se registró en un formulario. Los datos de los factores sociodemográficos, los hábitos y los comportamientos relacionados con las geohelmintiasis y la malaria, se recolectaron mediante dos cuestionarios: en el primero se les preguntaba a los directores de escuela sobre el censo de niños matriculados, las condiciones de acceso al agua y el saneamiento básico, y las actividades de desparasitación llevadas a cabo; en el segundo se recolectó la información sobre los factores de riesgo para geohelmintiasis en los niños (sexo, uso de calzado, geofagia, consumo de alimentos en la calle, lavado inadecuado de manos) y las variables socioeconómicas (disposición de excrementos, tipo de agua de consumo).

\section{Análisis estadístico}

La información recolectada se procesó en el programa Epi-Info $3.5 .1^{\circledR}$. La prevalencia de la infección causada por cualquiera de los geohelmintos se estimó con base en la de las infecciones individuales, utilizando el método descrito por Silva, et al. (12), y los respectivos intervalos de confianza (15). Se estimó la prevalencia comunitaria de infecciones de intensidad alta, moderada o leve causadas por cualquiera de los geohelmintos, de acuerdo con la fórmula propuesta por la OMS (12). En el análisis bivariado se utilizó la prueba de ji al cuadrado y el test exacto de Fisher con un nivel de alfa de 0,05. Para determinar la relación entre los factores de riesgo (consumo de agua impotable, consumo de agua de pozo, consumo de agua no envasada, defecación al aire libre y no usar calzado) y la presencia de cualquier parasitosis, se utilizó un modelo de regresión logística binaria ajustado por sexo y zonas eco-epidemiológicas en una etapa (pooled-data analysis). Se aplicó el test de Hosmer y Lemeshow, y se estimó la seudo $r$ al cuadrado de Nagelkerke, Cragg \& Uhler para determinar el nivel de ajuste del modelo con el programa SPSS $19^{\circledR}$.

\section{Aspectos éticos}

Se obtuvo el consentimiento informado por escrito de uno de los padres o cuidadores del niño. La Office for Human Research Protections del Department for Health and Human Services de los Estados Unidos (OHRP IRB № 0005660, FWA № 00010986) aprobó el protocolo del estudio, según consta en el acta número 4 de 2012. 


\section{Resultados}

Se recolectaron 1.352 muestras de heces en las 51 escuelas seleccionadas, de las cuales se descartaron 27 por tener una cantidad insuficiente - una consistencia inadecuada, es decir que se analizaron 1.325 muestras (93\% del tamaño estimado de la muestra). El $51 \%$ (676) de los escolares encuestados eran niñas y, el $49 \%$, niños. La media de la edad fue de 8,95 años y la mediana de 9, con una desviación estándar de $\pm 0,74$ años. La mediana de la talla fue de 129 $\mathrm{cm}$, con una desviación estándar de $\pm 6,4 \mathrm{~cm}$ y un rango intercuartílico entre $124 \mathrm{~cm}$ (percentil 25) y $134 \mathrm{~cm}$ (percentil 75). La mediana del peso fue de $27 \mathrm{~kg}$, con una desviación estándar de $\pm 6,1 \mathrm{~kg}$ y un rango intercuartílico entre $24 \mathrm{~kg}$ y $31 \mathrm{~kg}$. La mediana del índice de masa corporal fue de 16, con una desviación estándar de $\pm 3,03$ y un rango intercuartílico entre 14 y 18.

De los 152 niños cuyas muestras de sangre se sometieron a la prueba de detección de la malaria, $52,6 \%$ eran niñas y $47,4 \%$, niños. La media de edad en este grupo fue de 9,11 años y la mediana de 9, con una desviación estándar de $\pm 0,77$ años. La mediana de la talla fue de 131 $\mathrm{cm}$, con una desviación estándar de $\pm 5,6 \mathrm{~cm}$ y un rango intercuartílico entre $128 \mathrm{~cm}$ (percentil 25) y $133,8 \mathrm{~cm}$ (percentil 75). La mediana del peso fue de $29,2 \mathrm{~kg}$, con una desviación estándar de $\pm 6,5$ $\mathrm{kg}$ y un rango intercuartílico entre 25 y $31 \mathrm{~kg}$. La mediana del índice de masa corporal fue de 16,6, con una desviación estándar de $\pm 3,1$ y un rango intercuartílico entre 15 y 18,1.

La prevalencia de infección causada por cualquiera de los geohelmintos fue de 7,9\% ( $\left.\mathrm{IC}_{95 \%} 6,6-9,5\right)$ para todo el país, con incrementos según la zona eco-epidemiológica que oscilaron entre 2,6\% $\left(\mathrm{IC}_{95 \%}, 1,4-5,7\right)$ en la zona montañosa y $14,9 \%$ $\left(\mathrm{IC}_{95 \%}\right.$ 10,9-19,7) en la planicie costera. Al comparar las prevalencias de las zonas eco-epidemiológicas tomando como referencia la zona montañosa (que tuvo la prevalencia más baja), se observó un aumento a medida que el área geográfica se alejaba de esta y se acercaba a la planicie costera, en donde la prevalencia fue siete veces mayor, en tanto que en la depresión central fue cuatro veces mayor y en la cadena costera casi tres veces mayor (cuadro 2). La relación dosis-efecto entre el aumento de la prevalencia y las zonas ecoepidemiológicas fue estadísticamente significativa, con un ji al cuadrado de 30,86 ( $p<0,001)$.

La especie $T$. trichiura tuvo la mayor prevalencia, con 4,1\% (IC $\left.{ }_{95 \%} 3,1-5,3 \%\right)$, seguida de la infección por A. lumbricoides, con 2,7 \% (IC $\left.{ }_{95 \%} 2,0-3,7 \%\right)$ y por las uncinarias, con 1,8\% (IC $\left.{ }_{95 \%} 1,2-2,7 \%\right)$.

Se observó que la prevalencia de $A$. lumbricoides fue similar en los niños de 8 a 10 años de edad; la de $T$. trichiura fue mayor $(5,42 \%)$ en los niños y niñas de 8 años, y la de las uncinarias fue más elevada en los niños de 10 años de edad, con $2,67 \%$

Cuadro 2. Prevalencia de geohelmintiasis en escolares por zona eco-epidemiológica, edad y sexo, El Salvador, 2012

\begin{tabular}{|c|c|c|c|c|c|c|c|c|c|}
\hline Categoría de análisis & $\mathbf{n}$ & $\%$ & $\begin{array}{c}\text { Ascaris } \\
\text { Iumbricoides }\end{array}$ & $\%$ & $\begin{array}{l}\text { Trichuris } \\
\text { trichiura }\end{array}$ & $\%$ & Uncinarias & $\%$ & $\begin{array}{l}\text { Prevalencia } \\
\%\left(\mathrm{IC}_{95 \%}\right)^{\varepsilon}\end{array}$ \\
\hline \multicolumn{10}{|l|}{ Zona eco-epidemiológica } \\
\hline Cadena costera & 282 & 21,3 & 3 & 1,06 & 9 & 3,19 & 6 & 2,13 & $5,9 \quad(3,8-9,4)$ \\
\hline Cadena volcánica & 253 & 19,1 & 6 & 2,37 & 6 & 2,37 & 6 & 2,37 & $6,6 \quad(4,2-10,5)$ \\
\hline Depresión central & 290 & 21,9 & 16 & 5,52 & 9 & 3,10 & 5 & 1,72 & $9,4 \quad(6,5-13,2)$ \\
\hline Zona montañosa & 250 & 18,9 & 2 & 0,80 & 1 & 0,40 & 4 & 1,60 & $2,6 \quad(1,4-5,7)$ \\
\hline Planicie costera & 250 & 18,9 & 9 & 3,60 & 29 & 11,60 & 3 & 1,20 & $14,9(10,9-19,7)$ \\
\hline \multicolumn{10}{|l|}{ Edad (años) } \\
\hline 8 & 406 & 30,6 & 11 & 2,71 & 22 & 5,42 & 7 & 1,72 & $9,0 \quad(6,7-12,3)$ \\
\hline 9 & 582 & 43,9 & 17 & 2,92 & 23 & 3,95 & 8 & 1,37 & $7,6 \quad(5,7-10,0)$ \\
\hline 10 & 337 & 25,4 & 8 & 2,37 & 9 & 2,67 & 9 & 2,67 & $7,1 \quad(4,8-10,4)$ \\
\hline Total & 1.325 & 100,0 & 36 & 2.72 & 54 & 4,08 & 24 & 1,81 & $7,9 \quad(6,6-9,5)$ \\
\hline \multicolumn{10}{|l|}{ Sexo } \\
\hline Femenino & 676 & 51,0 & 19 & 2,81 & 26 & 3,85 & 15 & 2,22 & $8,1 \quad(6,3-10,4)$ \\
\hline Masculino & 649 & 49,0 & 17 & 2,62 & 28 & 4,31 & 9 & 1,39 & $7,7 \quad(5,9-10,0)$ \\
\hline Total & 1.325 & 100,0 & 36 & $\begin{array}{c}2,72 \\
\left(\mathrm{IC}_{95 \%} 2,0-3,7\right)\end{array}$ & 54 & $\begin{array}{c}4,1 \\
\left(\mathrm{IC}_{95 \%} 3,1-5,3\right)\end{array}$ & 24 & $\begin{array}{c}1,81 \\
\left(\mathrm{IC}_{95 \%}{ }^{1,2-2,7}\right)\end{array}$ & $7,9 \quad(6,6-9,5)$ \\
\hline
\end{tabular}

* Presencia de cualquier tipo de parásito (Ascaris lumbricoides, Trichuris trichuria o uncinaria)

\& Prevalencia de cualquier tipo de geohelminto calculada con la fórmula Path de Silva \& Hall (17):

$(a+t+u)-(a \times t+a \times u+t \times u)+(a \times t \times u) / 1,06$, donde $a=$ prevalencia de ascariasis (expresada como proporción), t=prevalencia de trichuriasis (expresada como proporción), y u=prevalencia de uncinariasis (expresada como proporción) 
(cuadro 2). La prevalencia de $A$. lumbricoides fue casi igual en ambos sexos, no así la de $T$. trichiura, que fue mayor en los niños, en tanto que la de las uncinarias fue mayor entre las niñas (cuadro 2).

La prevalencia de $A$. lumbricoides fue mayor en la zona de depresión central $(5,5 \%)$ y en la planicie costera $(3,6 \%)$; la prevalencia de $T$. trichiura fue mayor en la planicie costera $(11,6 \%)$ y entre los niños (12,9\%), en tanto que en las niñas fue menor $(10,4 \%)$. Por último, la prevalencia más elevada de uncinarias se dio en la cadena volcánica y costera con 2,4 y $2,1 \%$, respectivamente (cuadro 3 ).

La prevalencia de la infección de intensidad alta con cualquiera de las especies de geohelmintos fue de $0,3 \%(4 / 1.325)$, la moderada fue de $1,9 \%$ $(25 / 1.325)$ y la leve de 6,7 \% (85/1.325). La prevalencia de la infección de intensidad alta por A. lumbricoides y por uncinarias fue inferior al $1 \%(0,23$ y $0,08 \%$, respectivamente $)$, y no se registró ningún caso de infección de intensidad alta por $T$. trichiura.
En el análisis bivariado se encontró que los niños que defecaban al aire libre tuvieron tres veces más posibilidades de infección por geohelmintos que aquellos que no lo hacían; los que consumían agua de pozo tuvieron 2,7 veces más posibilidades de adquirir la infección que quienes consumían agua de otras fuentes; los que no usaban calzado de forma permanente tuvieron 2,3 veces más posibilidades que quienes lo usaban siempre, y los niños que no consumían agua potable tuvieron 2,2 veces más posibilidades que los que la consumían (cuadro 4). La regresión logística, que incluyó los factores de riesgo, el sexo y las zonas eco-epidemiológicas, mostró que los niños que defecaban al aire libre $(\mathrm{OR}=$ 2,$\left.2 ; I C_{95 \%} 1,1-4,2\right)$ y aquellos que no utilizaban calzado de forma permanente $\left(\mathrm{OR}=2,1 ; \mathrm{IC}_{95 \%}\right.$ $1,2-3,7)$, tuvieron mayores posibilidades de tener geohelmintiasis (cuadro 4) (seudo $r$ al cuadrado de Nagelkerke/Cragg \& Ulher: 0,098; prueba de Hosmer y Lemeshow: una etapa; ji al cuadrado: $10,474(p=0,233)$.

Cuadro 3. Prevalencia de geohelmintiasis por especie de parásito, zonas eco-epidemiológicas y sexo, El Salvador, 2012

\begin{tabular}{|c|c|c|c|c|c|c|c|c|c|c|}
\hline \multirow[t]{2}{*}{ Zona eco-epidemiológica } & \multicolumn{3}{|c|}{ A. lumbricoides } & \multicolumn{3}{|c|}{ T. trichiura } & \multicolumn{3}{|c|}{ Uncinaria } & \multirow[t]{2}{*}{ Total } \\
\hline & $\mathbf{F}$ & M & Subtotal & $\mathbf{F}$ & M & Subtotal & $\mathbf{F}$ & $\mathbf{M}$ & Subtotal & \\
\hline Cadena costera & 2,3 & 0,0 & 1,1 & 2,3 & 4,0 & 3,2 & 2,3 & 2,0 & 2,1 & 283 \\
\hline Cadena volcánica & 3,1 & 1,6 & 2,4 & 3,9 & 0,8 & 2,4 & 2,3 & 2,4 & 2,4 & 253 \\
\hline Depresión central & 3,5 & 7,5 & 5,5 & 2,1 & 4,1 & 3,1 & 2,8 & 0,7 & 1,7 & 290 \\
\hline Zona montañosa & 0,0 & 1,8 & 0,8 & 0,7 & 0,0 & 0,4 & 2,2 & 0,9 & 1,6 & 250 \\
\hline Planicie costera & 5,2 & 1,7 & 3,6 & 10,4 & 12,9 & 11,6 & 1,5 & 0,9 & 1,2 & 250 \\
\hline Total & 2,8 & 2,5 & 2,7 & 3,8 & 4,3 & 4,1 & 2,2 & 1,4 & 1,8 & 1.325 \\
\hline
\end{tabular}

Cuadro 4. Factores de riesgo relacionados con las geohelmintiasis en escolares, El Salvador, 2012

\begin{tabular}{|c|c|c|c|c|c|}
\hline \multirow[t]{2}{*}{ Factor de riesgo } & & \multicolumn{2}{|c|}{ Infección con geohelmintos } & \multirow{2}{*}{$\begin{array}{l}\text { Análisis bivariado } \\
\text { OR crudo }\left(\mathrm{IC}_{95 \%}\right)\end{array}$} & \multirow{2}{*}{$\begin{array}{l}\text { Regresión logística }^{(\mathrm{a})(\mathrm{b})} \\
\text { OR ajustado }\left(\mathrm{IC}_{95 \%}\right)\end{array}$} \\
\hline & & Sí & No & & \\
\hline \multirow[t]{2}{*}{ Sexo } & $M$ & 48 & 601 & 1,1 & 1,1 \\
\hline & $\mathrm{F}$ & 53 & 623 & $(0,7-1,6)$ & $(0,7-1,6)$ \\
\hline \multirow[t]{2}{*}{ No consume agua potable } & Sí & 43 & 311 & 2,2 & 1,3 \\
\hline & No & 58 & 913 & $(1,4-3,4)$ & $(0,5-3,4)$ \\
\hline \multirow[t]{2}{*}{ Consumo de agua de pozo } & Sí & 36 & 210 & 2,7 & 1,7 \\
\hline & No & 65 & 1.014 & $(1,7-4,1)$ & $(0,6-4,5)$ \\
\hline \multirow{2}{*}{ No consume agua envasada } & Sí & 99 & 1.189 & 1,5 & 1,5 \\
\hline & No & 2 & 35 & $(0,3-8,9)$ & $(0,3-7,9)$ \\
\hline \multirow[t]{2}{*}{ Defecación al aire libre } & Sí & 15 & 66 & 3,1 & 2,2 \\
\hline & No & 86 & 1.158 & $(1,7-5,6)$ & $(1,1-4,2)$ \\
\hline \multirow[t]{2}{*}{ No usa calzado siempre } & Sí & 86 & 869 & 2,3 & 2,1 \\
\hline & No & 15 & 355 & $(1,3-4,3)$ & $(1,2-3,7)$ \\
\hline
\end{tabular}

(a) Las variables incluidas en el modelo de análisis de regresión logística, fueron el sexo, no consumir agua potable, el consumo de agua de pozo, no consumir agua envasada, la defecación al aire libre y no usar calzado siempre.

(b): Resumen del modelo en una etapa = -2 logaritmo de verosimilitud $(658,861) ;$ r al cuadrado de Cox \& Snell $(0,41)$; seudo $r$ al cuadrado de Nagelkerke/ Cragg \& Ulher (0,098); prueba de Hosmer y Lemeshow: una etapa; ji al cuadrado $(10,474)(p=0,233)$ 
En el análisis bivariado de los factores de riesgo de la infección causada por las especies de geohelmintos, se encontró que la posibilidad de infección por $A$. lumbricoides $\left(\mathrm{OR}=3,9 ; \mathrm{IC}_{95 \%} 1,7\right.$ $9,3)$ era mayor cuando los niños defecaban al aire libre y consumían agua de pozo. En el caso de $T$. trichiura, la posibilidad de infección fue mayor en niños que no consumían agua potable $(\mathrm{OR}=2,3$; $\left.\mathrm{IC}_{95 \%} 1,3-3,9\right)$ sino agua de pozo (OR=3,2; $\mathrm{IC}_{95 \%}$ $1,8-5,6)$. Los factores asociados con la infección por uncinarias, fueron el mal lavado de manos $\left(\mathrm{OR}=3,1 ; \mathrm{IC}_{95 \%}, 1,1-9,1\right)$ y no usar calzado de forma permanente (OR=9,0; IC $\left.{ }_{95 \%} 1,2-180,7\right)$, aunque el tamaño del intervalo de confianza en este último caso fue bastante amplio.

Las 152 muestras analizadas para la detección de malaria mediante la prueba rápida y la PCR, fueron negativas en el examen por microscopía (gota gruesa y frotis de espesor delgado). La concordancia de $100 \%$ en las tres técnicas de diagnóstico utilizadas, confirmó la ausencia de casos asintomáticos de malaria en la muestra del estudio.

\section{Discusión}

La prevalencia de infecciones por geohelmintos encontrada en este estudio difiere de algunos datos publicados sobre El Salvador en años anteriores. Según un estudio en una muestra de niños con síndrome diarreico publicado en 1988 (16), la prevalencia de T. trichiura era de $31 \%$, la de $A$. lumbricoides, de $18 \%$, y la de uncinarias, de $0 \%$. Debe señalarse que aunque dicho estudio tiene un sesgo de selección, pues no se llevó a cabo en niños sanos y el lavado intestinal por la diarrea pudo haber afectado la cantidad de huevos de geohelmintos detectables, es uno de los pocos reportados en la literatura científica sobre el caso de El Salvador. En otro estudio publicado en el 2004, se reportó una prevalencia de uncinariasis entre el 0,0 y el $9,9 \%$ (17). En el presente estudio la probabilidad de infección por geohelmintos en las niñas fue casi dos veces mayor que en los varones ( $A$. lumbricoides y uncinarias) y, aunque la diferencia no fue estadísticamente significativa, podrían existir factores actitudinales, culturales o biológicos dependientes del sexo que se relacionen con el riesgo de infección. Sin embargo, serán necesarios otros estudios para evaluar esta hipótesis.

Aunque se encontró que en 17 (33,3\%) de los centros escolares del estudio los niños habían recibido medicamentos antiparasitarios en los tres meses anteriores, es importante resaltar que se encontraron escolares con parásitos, lo que indica que más allá de la disponibilidad y el acceso al tratamiento, las condiciones sociales y ambientales inadecuadas tienen un efecto importante en la reinfección, principalmente en el área rural (18).

En otros estudios se han reportado valores de prevalencia mayores en poblaciones que tienen dificultades de acceso al saneamiento básico o que viven en extrema pobreza. En un estudio publicado en 2006, sobre la asociación entre el sistema de saneamiento y la prevalencia de helmintiasis en la población rural de El Salvador, se reportó una prevalencia de $8,0 \%$ de $A$. lumbricoides, de $20,9 \%$ de $T$. trichiura y de $22,3 \%$ de uncinarias (19). En dos estudios de tesis llevados a cabo por estudiantes de la Facultad de Medicina de la Universidad de El Salvador en 1996 y 1998, se reportaron prevalencias de geohelmintiasis cercanas al $50 \%$ en municipios con pobreza extrema $(20,21)$. Entre el 2002 y el 2012, el Ministerio de Salud y el Ministerio de Educación adelantaron campañas nacionales de desparasitación dos veces al año mediante el programa de escuelas saludables, que beneficiaron a una población de 500.000 a 800.000 escolares, con excepción de las campañas de 2010 y 2012, en las que se reportó un número de desparasitados de 106.000 y 366.000 estudiantes, respectivamente (22).

Además, entre el 2006 y el 2009, varias organizaciones no gubernamentales reportaron a la OPS/OMS diversas actividades de desparasitación masiva en el país, con un cubrimiento de 723.000 escolares en el 2009. La regularidad de dichas actividades podría explicar parcialmente los niveles de prevalencia inferiores al $20 \%$ en este estudio. El Salvador sería uno de los países que ha administrado el tratamiento preventivo para el control de las geohelmintiasis durante más de cinco años, intervención que, según las guías de la OMS (23), podría ajustarse de acuerdo con los niveles de prevalencia encontrados en este estudio. Por ejemplo, en las zonas eco-epidemiológicas en donde se encontraron prevalencias entre $1 \% 0$ más y menos de $10 \%$ (zona montañosa, cadena volcánica, cadena costera y depresión central), el tratamiento preventivo podría hacerse una vez cada dos años, mientras que en las zonas con prevalencias entre $10 \%$ o más y menos de $20 \%$ (planicie costera), se continuaría con una ronda de tratamiento preventivo anual. Sin embargo, es necesario mantener la vigilancia de la prevalencia y la intensidad de la infección en el país y por 
zonas eco-epidemiológicas, para vigilar posibles cambios en estos dos indicadores en razón de las condiciones sociales y económicas, en especial, lo referente al acceso a agua segura y el saneamiento básico. Según la Encuesta de Hogares de Propósitos Múltiples llevada a cabo en el 2010 en El Salvador, el $83 \%$ de los hogares tenía acceso al agua por tubería (93\% en las áreas urbanas y $64 \%$ en las rurales) y persistía un gran déficit en el acceso a la infraestructura sanitaria (disposición de excrementos y aguas residuales) ya que según la Administración Nacional de Acueductos y Alcantarillados, solo 81 (31\%) de los 262 municipios contaban con servicios adecuados de alcantarillado, y el $43 \%$ de las viviendas urbanas y el $98 \%$ de las rurales no estaban conectadas a la red de alcantarillado. Además, se reportaba que el $14 \%$ de la población rural no disponía de servicio sanitario alguno (24).

Las diferencias de prevalencia de geohelmintiasis entre las zonas eco-epidemiológicas del estudio, ratificarían el efecto de las condiciones ambientales y los factores socioeconómicos en la transmisión (25). Se sabe que los huevos de T. trichiura requieren condiciones de suelo arenoso, y temperatura y humedad elevadas, para producir las larvas (26), lo que explicaría que en el presente estudio este haya sido el geohelminto con mayor prevalencia nacional $(4,1 \%)$, sobre todo en la planicie costera, seguido de $A$. lumbricoides, con una prevalencia nacional de 2,7\%, primordialmente en la depresión central, cuya temperatura promedio es de $30^{\circ} \mathrm{C}$, independientemente del tipo de suelo, lo que podría ser un factor determinante en su distribución. Las uncinarias requieren de suelo húmedo con escasa radiación solar, humedad constante y vegetación herbácea, condiciones que se encuentran en abundancia en las cadenas volcánica y costera. La detección de uncinarias debe hacerse inmediatamente después de la toma de las muestras, lo cual no fue posible en este estudio por dificultades logísticas a nivel local, por lo que debieron analizarse de 24 a 48 horas después de su recolección, y explicaría su baja detección $(1,8 \%)(27,28)$.

Entre las características epidemiológicas más llamativas de las infecciones por helmintos en los humanos, se encuentran su agregación en las comunidades, la predisposición de los individuos a infecciones intensas (o leves), la rápida reinfección después del tratamiento preventivo y los perfiles de intensidad relacionados con la edad (alta en los niños y adultos mayores, con excepción de las uncinarias). La tasa de reinfección depende de varios factores, entre los que se encuentran la especie de helminto y su ciclo de vida (los helmintos que tienen vida corta reinfectan más rápidamente), la intensidad de la transmisión en la comunidad, y la eficacia y cobertura del tratamiento (29).

Aunque estos factores pueden tener relación con la intensidad de la infección, en este estudio solo el $0,3 \%$ de los niños tenía infecciones de intensidad alta, probablemente por el carácter sistemático de las intervenciones para el control de las geohelmintiasis, lo cual coincide con los lineamientos de la OMS de reducir dichas infecciones a menos del $1 \%$ mediante intervenciones rutinarias (12). Los niños en edad escolar generalmente presentan las infecciones parasitarias de mayor intensidad, lo que afecta negativamente su salud general, nutrición, desarrollo cognitivo, aprendizaje, acceso a la educación y desempeño académico (30).

Los factores de riesgo, como la defecación al aire libre, el consumo de agua de pozo o no potable y no usar calzado, fueron los más asociados con la prevalencia de geohelmintiasis en este estudio. Es bien conocido que los factores relacionados con el huésped y con el medio ambiente inciden en el riesgo de adquisición o persistencia de las infecciones por helmintos de intensidad alta. El trabajo en la agricultura y la minería, así como el hacinamiento y los hábitos de higiene inadecuados, influyen en la prevalencia y la intensidad de la infección por helmintos en una comunidad. La presencia de helmintos está íntimamente relacionada con la pobreza, el saneamiento deficitario y la falta de agua potable lo cual implica que la desparasitación rutinaria no basta para reducir la prevalencia y la intensidad de la infección, sino que deben diseñarse intervenciones con todos los sectores pertinentes para ampliar los grupos de población que reciben el tratamiento antiparasitario (preescolares, mujeres a partir del primer trimestre de embarazo y trabajadores agrícolas o mineros), y mejorar el acceso al agua potable y a instalaciones mejoradas de saneamiento básico.

El Ministerio de Salud de El Salvador debe revisar y ajustar su plan de acción para el control de las geohelmintiasis con base en los resultados del presente estudio, e introducir el componente educativo para promover los hábitos higiénicos adecuados. Se recomienda, igualmente, implementar la vigilancia centinela de los indicadores parasitológicos para geohelmintos, con el fin de registrar los avances y afianzar los resultados de cumplimiento de la meta de control, es decir, una prevalencia de infección y 
de infecciones de intensidad alta de menos del $1 \%$, cumpliendo así con los parámetros establecidos por la OMS para los programas de control que han aplicado el tratamiento preventivo durante cinco o seis años consecutivos.

En cuanto a las muestras sanguíneas para el diagnóstico de la malaria, se registró $100 \%$ de concordancia entre los análisis por microscopía, las técnicas inmunológicas rápidas y la prueba de PCR, pues todas arrojaron resultados negativos. Esta información es útil para solicitar un futuro proceso de verificación de la eliminación de la malaria en el país, ya que la tasa de morbilidad por paludismo fue de 0,21 casos por 100.000 habitantes en 2012 (31). Para los países que se encuentran en la fase previa a la eliminación, la OPS/OMS ha establecido un índice de menos de $5 \%$ de láminas positivas en los casos sospechosos y de menos de 0,5 casos por 1.000 personas en riesgo en un año; El Salvador ya cumple con parte de dichos criterios y debe centrarse en el cumplimiento de los que faltan para alcanzar la fase de eliminación (32).

En el presente estudio no se encontraron portadores de Plasmodium spp. en los escolares estudiados. Una de las debilidades del estudio fue el tamaño de la muestra, el cual fue insuficiente para concluir que no existe transmisión autóctona en estos cinco municipios, por lo que se recomienda hacer estudios con mayor alcance geográfico, como una encuesta de población que contemple el cuadro hemático y cuente con métodos de diagnóstico molecular para un análisis más sensible que detecte a los portadores asintomáticos. Una encuesta así debe incluir otros grupos de edad para tener un conocimiento más completo de la transmisión de la malaria en el país.

Aunque los índices de malaria en El Salvador cumplen con parte de los requisitos de la eliminación, el riesgo de que se restablezca la transmisión en las áreas libres de malaria sigue siendo latente. Cabe mencionar que los casos de malaria en El Salvador provienen de Sudán y Guinea Ecuatorial, así como de países vecinos como Guatemala, Honduras y Nicaragua, lo que incrementa el riesgo de la transmisión autóctona de casos a partir de casos importados, así como de la aparición de $P$. falciparum en el país. Por ello, es necesario mantener los logros alcanzados y avanzar en el cumplimiento de los otros criterios establecidos por la OPS/OMS para transitar hacia la eliminación, incluidos el fortalecimiento del sistema de vigilancia, la colaboración de los sectores públicos y privados de la salud, así como el mantenimiento y fortalecimiento de la capacidad local para la adecuada detección de casos, su diagnóstico y tratamiento oportuno y de calidad, y para el manejo de los focos.

En el periodo del estudio, el Ministerio de Salud de El Salvador formuló el Plan de Control y Eliminación de las Enfermedades Infecciosas Desatendidas, en el cual se incluyó el control de las geohelmintiasis y la malaria. Este plan prevé un manejo intersectorial de los factores determinantes de estas enfermedades, por lo que constituye una oportunidad para seguir administrando el tratamiento preventivo de las geohelmintiasis y las acciones tendientes a la eliminación de la malaria y, además, involucrar a todos los sectores en los esfuerzos para reducir las brechas de desarrollo, en especial lo que tiene que ver con el acceso a una vivienda adecuada, a agua segura y al saneamiento básico, con énfasis en la disposición adecuada de excretas, así como en la educación.

\section{Agradecimientos}

Al personal docente de los centros escolares, a las familias y alumnos participantes; al personal de los equipos comunitarios de salud familiar, a Región de Salud, y a todo el personal de salud que participó en las diferentes etapas de la investigación, así como al Laboratorio de Referencia de la Secretaría de Salud de Honduras, por su apoyo en el análisis de muestras.

\section{Conflicto de intereses}

La presente investigación no presentó conflicto de intereses ni conflictos éticos con ninguna institución, persona o entidad relacionada con el tema.

\section{Financiación}

Los Ministerios de Salud y de Educación de El Salvador, los Programas Regionales de Malaria y de Enfermedades Infecciosas Desatendidas de la OPS/OMS, y el Proyecto de Prevención y Control de Enfermedades cofinanciado por la OPS/OMS y el Departamento de Asuntos Exteriores, Comercio y Desarrollo del Canadá (anterior Agencia Canadiense para el Desarrollo InternacionalACDI), aportaron a la financiación del estudio.

\section{Referencias}

1. World Health Organization/Pan American Health Organization. Epidemiological profile of neglected diseases and other infections related to poverty in Latin American and the Caribbean. Washington, D.C.: PAHO; 2009. Fecha de 
consulta: 30 de septiembre de 2013. Disponible en: http:// www2.paho.org/hq/dmdocuments/2009/nds-epi-profiles.pdf

2. World Health Organization. Prevention and control of schistosomiasis and soil-transmitted helminthiasis. Report of a WHO expert committee. Technical Report Series № 912. Geneva: WHO; 2002.

3. Organización Panamericana de la Salud. Resolución CD49.R9. Eliminación de las enfermedades desatendidas y otras infecciones relacionadas con la pobreza. $49^{\circ}$ Consejo Directivo. Washington, D.C.: OPS; 2009. Fecha de consulta: 30 de enero de 2014. Disponible en: http://new.paho.org/hq/ dmdocuments/2009/CD49.R19\%20(Eng.).pdf.

4. Saboyá MI, Catalá L, Nicholls RS, Ault SK. Update on the mapping of prevalence and intensity of infection for soiltransmitted helminth infections in Latin America and the Caribbean: A call for action. PLoS Negl Trop Dis. 2013;7: e2419. http://dx.doi.org/10.1371/journal.pntd. 0002419

5. Ministerio de Educación. Estadísticas Censo Escolar. 2009-2011. Fecha de consulta: 20 de febrero de 2014. Disponible en: https://www.mined.gob.sv/index.php/institucion/ marco/historia/6589-censo-final-2013.html.

6. World Health Organization. Soil-transmitted helminthiases: Estimates of the number of children needing preventive chemotherapy and number treated, 2009. Wkly Epidemiol Rec. 2011:86;257-68.

7. Organización Panamericana de la Salud. Resolución CD51.11. Estrategia y plan de acción sobre la malaria. 51 ${ }^{\circ}$ Consejo Directivo, Washington DC: OPS-OMS: 2011. Fecha de consulta: 20 de febrero de 2014. Disponible en: http://www.paho.org/hq/index.php?option=com_docman \&task $=$ doc_download\&gid $=14504 \&$ ltemid $=9$.

8. World Health Organization. World Malaria Report 2013. Fecha de consulta: 10 de noviembre de 2014. Disponible en: http://www.paho.org/HQ/index.php?option=com_docman \&task=doc_details\&gid=23821\&ltemid=270\&lang=es.

9. Hotez PJ, Molyneux DH, Fenwick A, Kumaresan J, Sachs SE, Sachs JD, et al. Control of neglected tropical diseases. N Eng J Med. 2007;357:1018-27. http://dx.doi.org/10.1056/ NEJMra064142

10. Organización Panamericana de la Salud. Control de helmintiasis en niños en edad escolar: una guía para gerentes de programas de control. Ginebra. OPS; 2009. p. 29.

11. World Health Organization. Basic laboratory methods in medical parasitology. Geneva: WHO; 1991. p. 25-8.

12. World Health Organization. Helminth control in school-age children: A guide for managers of control programmes. 2nd edition. Geneva: WHO; 2011.

13. De Lamballerie $\mathbf{X}$, Zandotti $\mathbf{C}$, Vignoli $\mathbf{C}$, Bollet $\mathbf{C}$, de Micco P. A one-step microbial DNA extraction method using "Chelex 100" suitable for gene amplification. Res Microbiol. 1992:143:785-90.

14. Singh B, Bobogare A, Cox-Singh J, Snounou G, Abdullah MS, Rahman HA. A genus- and species-specific nested polymerase chain reaction malaria detection assay for epidemiologic studies. Am J Trop Med Hyg. 1999:60: 687-92.
15. Newcombe RG. Two-sided confidence intervals for the single proportion: Comparison of seven methods. Stat Med. 1998:17;857-72. http://dx.doi.org/10.1002/(SICI)10970258(19980430)17:8<857::AID-SIM777>3.0.CO;2-E

16. Reinthaler FF, Lick G, Klem G, Mascher F, Sixl W. Intestinal parasites in children with diarrhea in El Salvador. Geogr Med. 1998:18:175-80.

17. Brooker S, Bethony J, Hotez PJ. Human hookworm infection in the 21st century. Adv Parasitol. 2004;58:197288. http://dx.doi.org/10.1016/S0065-308X(04)58004-1

18. Smith HM, DeKaminsky RG, Niwas S, Soto RJ, Jolly PE. Prevalence and intensity of infections of Ascaris lumbricoides and Trichuris trichiura and associated socio-demographic variables in four rural Hondurean communities. Mem Inst Oswaldo Cruz. 2001;96:303-14. http://dx.doi.org/10.1590/ S0074-02762001000300004

19. Corrales LF, Izurieta R, Moe CL. Association between intestinal parasitic infection and type of sanitation system in rural El Salvador. Trop Med Int Health. 2006:11:1821-31. http://dx.doi.org/10.1111/j.1365-3156.2006.01737.x

20. Ángel-Belloso RO, Zaldaña SB, Bran EE. Frecuencia de parasitismo intestinal en la población de cuatro comunidades rurales del departamento de La Paz. Comparación de los resultados parasitológicos obtenidos por un grupo de laboratoristas clínicos con los de un laboratorio de referencia (tesis). San Salvador: Universidad de El Salvador; 1996.

21. Paredes RE. Parasitismo intestinal en la población estudiantil de la Escuela Rural Mixta Unificada del caserío El Cajón, Huizúcar, La Libertad (tesis). San Salvador: Universidad de El Salvador; 1998.

22. World Health Organization. Soil-transmitted helminthiasis. Countries $x$ indicators. Fecha de consulta: 16 de enero de 2014. Disponible en: http://www.who.int/neglected diseases/preventive_chemotherapy/sth/db/index.html?unit $\mathrm{s}=$ minimal\&region=all\&country=slv\&countries=slv\&year=all.

23. World Health Organization. Preventive chemotherapy in human helminthiasis: Coordinated use of anthelminthic drugs in control interventions: A manual for health professionals and programme managers. Geneva: WHO; 2006. p. 41.

24. Organización Panamericana de la Salud. La salud en las Américas. Capítulo El Salvador. 2012. Fecha de consulta: 16 de enero 2014. Disponible en: http://www.paho.org/ saludenlasamericas/index.php?option=com_content\&view $=$ article\&id $=36 \&$ ltemid $=35 \&$ lang $=$ es.

25. World Health Organization. Global plan to combat neglected tropical diseases 2008-2015. Geneva: WHO; 2007. p. 9-10.

26. Miyazaki I. Helminthic zoonoses. Fukuoka: International Medical Foundation of Japan; 1991. p. 447-50.

27. Dacombe RJ, Crampin AC, Floyd S, Randall A, Ndhlovu $\mathbf{R}$, Bickle $\mathbf{Q}$, et al. Time delays between patient and laboratory selectively affect accuracy of helminth diagnosis. Trans R Soc Trop Med Hyg. 2007;101:140-5. http://dx.doi. org/10.1016/j.trstmh.2006.04.008

28. World Health Organization. Bench aids for the diagnosis of intestinal parasites. Geneva: WHO; 1994. 
29. Hotez PJ, Bundy DA, Beegle K, Brooker S, Drake L, de Silva $\mathbf{N}$, et al. Helminth infections: Soil-transmitted helminth infections and schistosomiasis. En: Jamison DT, Breman JG, Measham AR, Alleyne G, Claeson M, Evans DB, et al., editors. Disease control priorities in developing countries. 2nd edition. New York: The World Bank and Oxford University Press; 2006. p. 467-82.

30. World Bank. School Deworming at a Glance. 2003. Fecha de consulta: 10 de febrero de 2014. Disponible en: http:// siteresources.worldbank.org/INTPHAAG/Resources/AAG DewormingEng110603.pdf.
31. Ministerio de Salud de El Salvador. Informe de vigilancia y control de enfermedades transmitidas por vectores: dengue, malaria, Chagas y leishmaniasis. San Salvador: Ministerio de Salud; 2012. p. 17.

32. Organización Panamericana de la Salud. Guía para la reorientación de los programas de control de la malaria con miras a la eliminación. Washington, D.C.: OPS; 2011. Fecha de consulta: 10 de septiembre de 2013. Disponible en: http://www2.paho.org/hq/dmdocuments/2011/Guiareorient-progr-control-malaria-eliminacion.pdf. 